\title{
Oncostatin M, an Inflammatory Cytokine Produced by Macrophages, Supports Intramembranous Bone Healing in a Mouse Model of Tibia Injury
}

Pierre Guihard, Marie-Astrid Boutet, Bénédicte Brounais-Le Royer, Anne-Laure Gamblin, Jérôme Amiaud, Audrey Renaud, Martine Berreur, Françoise Rédini, Dominique Heymann, Pierre Layrolle, and Frédéric Blanchard

From INSERM, UMR 957, Ligue Team 2012, Nantes; and the Pathophysiology of Bone Resorption Laboratory and Therapy of Primary Bone Tumors, Medicine Faculty, Université de Nantes, Nantes Atlantique Universités, Nantes, France

Accepted for publication

November 12, 2014.

Address correspondence to Frédéric Blanchard, Ph.D., INSERM-UN UMR 957, 1 rue Gaston Veil, F44035 Nantes, France. E-mail: frederic blanchard@univ-nantes.fr.

\begin{abstract}
Different macrophage depletion strategies have demonstrated a vital role of macrophages in bone healing, but the underlying molecular mechanisms are poorly understood. Here, with the use of a mouse model of tibia injury, we found that the cytokine oncostatin M [OSM or murine (m)OSM] was overexpressed during the initial inflammatory phase and that depletion of macrophages repressed mOSM expression. In $\mathrm{Osm}^{-/-}$mice, by micro-computed tomography and histology we observed a significant reduction in the amount of new intramedullar woven bone formed at the injured site, reduced number of 0sterix ${ }^{+}$osteoblastic cells, and reduced expression of the osteoblast markers runt-related transcription factor 2 and alkaline phosphatase. In contrast, osteoclasts were normal throughout the healing period. One day after bone injury, Stat3, the main transcription factor activated by MOSM, was found phosphorylated/activated in endosteal osteoblastic cells located at the hedge of the hematoma. Interestingly, we observed reduced activation of Stat3 in $\mathrm{Ssm}^{-/-}$mice. In addition, mice deficient in the mOSM receptor $\left(\mathrm{Ssmr}^{-/-}\right)$also had reduced bone formation and osteoblast number within the injury site. These results suggest that mOSM, a product of macrophages, sustains intramembranous bone formation by signaling through 0smr and Stat3, acting on the recruitment, proliferation, and/or osteoblast differentiation of endosteal mesenchymal progenitor cells. Because bone resorption is largely unaltered, OSM could represent a new anabolic treatment for unconsolidated bone fractures. (Am J Pathol 2015, 185: 765-775; http://dx.doi.org/10.1016/j.ajpath.2014.11.008)
\end{abstract}

With an aging population, fractures are becoming a common clinical problem, especially fractures associated with nonunion or delayed healing. ${ }^{1}$ Each year, $2 \%$ of the population would be affected after a trauma, infection, cancer, or orthopedic implant, for example. Antiresorptive agents such as bisphosphonates efficiently reduce the risk of fracture through inhibition of bone resorption by osteoclasts, but subsequently bone formation by osteoblasts is suppressed. ${ }^{2}$ Indeed, only a few truly anabolic bone therapies are available to induce bone reconstruction. Parathyroid hormone (PTH) or bone morphogenetic proteins are known to improve fracture healing in animal models, but their complexity of action on both bone formation and resorption and their relative inefficacy in clinical trials highlights our need for new anabolic agents. $1,3,4$
Fracture healing is a multistep process that involves recruitment of mesenchymal progenitor cells and differentiation into osteoblasts to form a hard callus that is secondarily remodeled by osteoclasts. ${ }^{4,5}$ Different types of bone reconstruction can be observed, through either endochondral ossification, whereby a cartilaginous model is first produced and then mineralized, or intramembranous

Supported by INSERM, the region Pays de la Loire (CIMATH2 project), the French Agency for Biomedicine, and fellowship from le French Ministry of Research (P.G.).

P.G. and M.-A.B. contributed equally to this work.

Disclosures: None declared.

Current address of P.G., Division of Cardiology, David Geffen School of Medicine at UCLA and Molecular Biology Institute, University of California, Los Angeles, CA. 

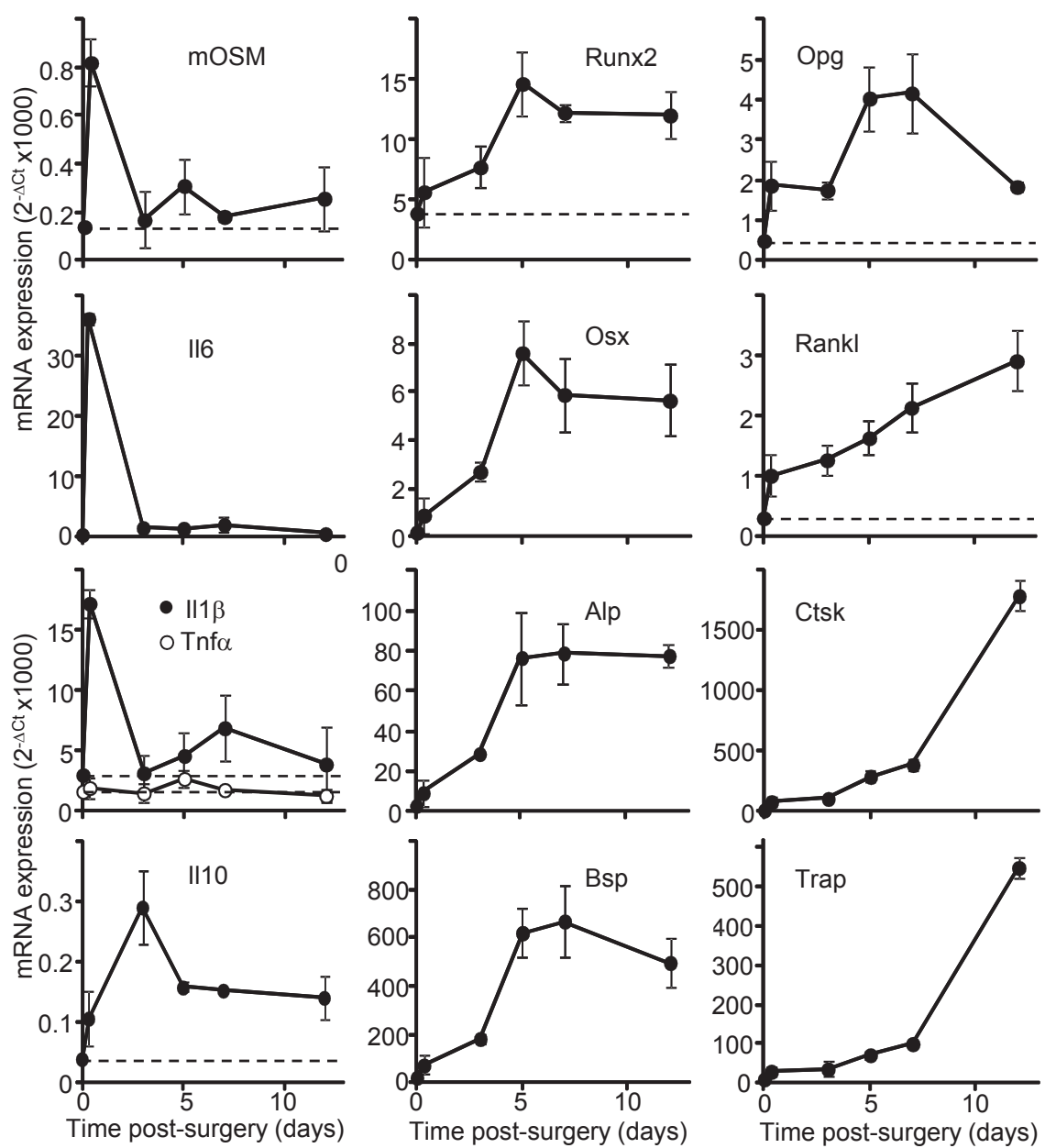

Figure 1 mOSM is expressed in the bone injury site. mRNA expression of indicated genes was analyzed by quantitative real-time RT-PCR in the mouse tibial injury model at different times after surgery. Gapdh was used as an invariant control. Similar results were obtained in another independent experiment. The dotted lines represent the expression amount without bone injury. Data
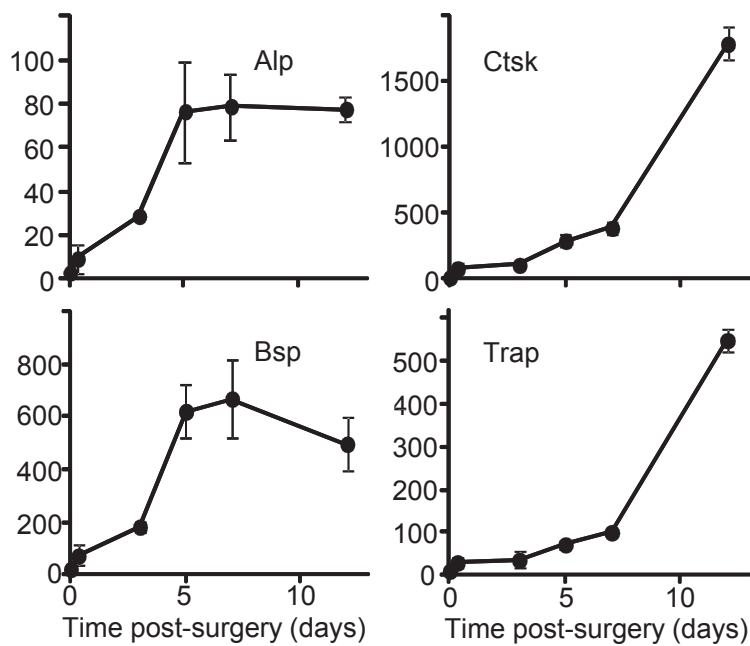
are expressed as means \pm SD. $n=3$ mice for each time point. Alp, alkaline phosphatase; Bsp, bone sialoprotein; Ctsk, cathepsin K; Gapdh, glyceraldehyde-3-phosphate dehydrogenase; 0pg, osteoprotegerin; mOSM, murine oncostatin $\mathrm{M}$; Osx, osterix; Rankl, receptor activator of NF- $\mathrm{KB}$ ligand; Runx2, runt related transcription factor 2; Tnf- $\alpha$, tumor necrosis factor $\alpha$; Trap, tartrate-resistant acid phosphatase.

ossification, whereby the bone matrix is directly formed. ${ }^{4,5}$ Noncritical bone fractures heal mainly through intramembranous ossification, but, in the case of nonunion and unconsolidated fractures, endochondral ossification is largely involved. More recently, it has become clear that inflammation plays a key role in fracture repair. Indeed, during the first few days, a hematoma is formed with infiltration of inflammatory cells and secretion of cytokines and growth factors. ${ }^{6}$ In murine models, tumor necrosis factor $\alpha$ and IL- 6 are produced at the fracture site within 24 hours of injury and are implicated in the recruitment and differentiation of mesenchymal progenitor cells to promote bone healing. ${ }^{1,7}$ However, inflammation must be tightly controlled and resolved, with a key role here for antiinflammatory cytokines such as IL-10. ${ }^{8}$

In the field of osteoimmunology, the innate and adaptive immune systems were shown to regulate the bone tissue, and there is an intense crosstalk between bone and immune cells. In the absence of lymphocytes, fracture healing is accelerated, whereas depletion of macrophages prevents intramembranous bone healing. ${ }^{4,8}$ Macrophages thus appear to have a vital role in the regulation of bone formation during inflammation and bone injury. With the use of a high-throughput approach in culture models, we previously demonstrated that monocytes/ macrophages secrete a major cytokine of the IL-6 family, oncostatin M [OSM or murine (m)OSM], to promote osteogenesis in mesenchymal stem cells (MSCs). ${ }^{10}$ These results were confirmed in two other studies. ${ }^{11,12}$ In mice models, injection of recombinant mOSM enhances calvarial thickness, and overexpression of $\mathrm{mOSM}$ in long bone induces woven bone formation that resembles intramembranous bone healing. ${ }^{10,13}$ The bone phenotype of $\mathrm{Osm}^{-1-}$ mice was not yet published, but mOSM receptor (Osmr)-deficient mice were previously analyzed. ${ }^{13} \mathrm{Osmr}^{-/-}$mice have reduced number of osteoblasts and osteoclasts, resulting in osteopetrotic bones. Unfortunately, these mice were not used to assess the role of the Osm-Osmr signaling in fracture healing.

Here, with the use of a noncritical injury model in tibia of mice, we show that mOSM is expressed early during the inflammatory phase mainly by macrophages and that $O s m$ or Osmr deficiency leads to delayed bone healing.

\section{Materials and Methods}

\section{Murine Model of Bone Injury and Knockout Mice}

All research involving animals was conducted according to the institutional guidelines and was approved by the French ethical committee CEEA.PdL.06 and by local veterinary 

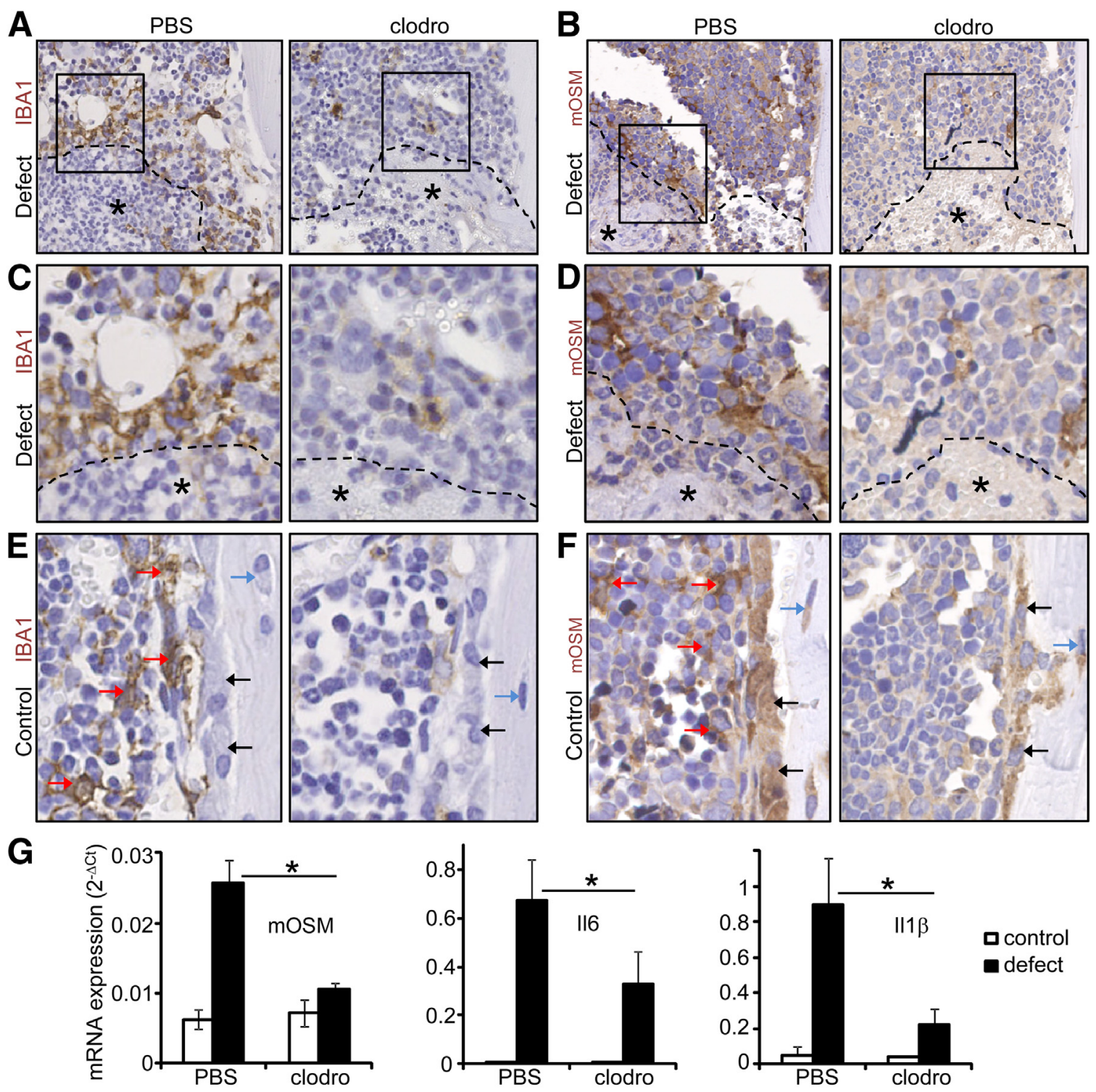

Figure 2 Macrophage depletion represses mOSM expression. A-F: Mice were treated with the macrophage-depleting agent clodro or control PBS liposome. Eight hours after tibial injury (dotted areas) animals were sacrificed, and tibial sections were stained for IBA1 (pan-macrophages in brown) (A, C, and E) or m0SM (B, D, and F). Representative images are shown in the bone injury site (defect) and the contralateral noninjured leg (control). $\mathbf{C}$ and $\mathbf{D}$ are enlarged images of the boxed areas in A and B. Asterisks indicate hematoma with eventual bone debris; black arrows, endosteal osteoblasts; blue arrows, osteocytes; red arrows, macrophages. G: mOSM, Il6, and Il1 $\beta$ mRNA expression was analyzed by quantitative real-time RT-PCR 8 hours after surgery. Hprt was used as an invariant control. Data are expressed as means \pm SD. $n=3$ mice per group $(\mathbf{A}-\mathbf{F}) ; n=4$ mice in PBS + control, clodro + control, and PBS + defect groups $(\mathbf{G}) ; n=3$ mice in the clodro + defect group $(\mathbf{G}) .{ }^{*} P<0.05$ versus PBS. Original magnification: $\times 20(\mathbf{A}$ and $\mathbf{B}) ; \times 40(\mathbf{C}-\mathbf{F})$. Clodro, clodronate liposome; Hprt, hypoxanthine-guanine phosphoribosyltransferase; IBA1, ionized calcium-binding adapter molecule 1; mOSM, murine oncostatin M; PBS, phosphate-buffered saline.

services (license no. D-44015) and was performed under the supervision of authorized investigators. The generation and genotyping of $\mathrm{Osm}$ and $\mathrm{Osmr}^{-1-}$ mice, backcrossed onto C57BL/6, was described previously ${ }^{14,15}$ and were kindly provided by Dr Atsushi Miyajima (University of Tokyo, Japan). All experiments were conducted with wild-type (WT) and $\mathrm{Osm}^{-1-}$ (or $\mathrm{Osmr}^{-1-}$ ) littermates obtained from our breeding colony by using heterozygous breeding pairs. Mice were housed in pathogen-free facilities and under conditions controlled for light (12 hours light/dark cycle), temperature $\left(22^{\circ} \mathrm{C}\right.$ to $\left.25^{\circ} \mathrm{C}\right)$, and humidity ( $50 \%$ to $\left.60 \%\right)$.
For noncritical bone injury, 7- to 8-week-old mice were anesthetized by inhalation of an isoflurane-air mixture ( $2 \%, 0.2 \mathrm{~L} /$ minute) and injected with $20 \mu \mathrm{g} / \mathrm{kg}$ Buprecare (Animalcare, Dunnington, UK). Hair was removed from the left hindlimb, and an incision was made in the skin over the medial part of the proximal tibia. Soft tissue was removed, and a hole $(1.0 \mathrm{~mm}$ in diameter) was created through cortical and medullar bone (the opposite side of cortical bone was preserved) by using a 19-gauge needle attached to an electric drill (Surgic XT; NSK, Paris, France). Because no differences were observed in bone 
healing between male and female mice, both sexes were used indifferently.

\section{Macrophage Depletion Using Clodronate Liposome}

Mice were treated daily by intraperitoneal injection of 10 $\mu \mathrm{L} / \mathrm{g}$ clodronate or control phosphate-buffered saline (PBS) liposomes (ClodronateLiposomes.com; Haarlem, The Netherlands) as described previously., ${ }^{4,16}$ After 3 days, the bone injury was performed, and mice received an additional $50 \mu \mathrm{L}$ of intradefect injection of clodronate or PBS liposomes. Animals were sacrificed 8 hours later for immunohistochemistry and real-time PCR analysis.

\section{microCT}

Bone architecture was analyzed at the indicated time on anesthetized animals [8\% xylazine (Rompun; Bayer, Deerfield, IL) and 13\% ketamine (Imalgène 500; Merial, London, UK) in PBS; $100 \mu \mathrm{L} / 10 \mathrm{~g}$ ] by using the high-resolution SkyScan-1076 X-ray micro-computed tomography (microCT) system for small animal imaging (SkyScan, Kartuizersweg, Belgium). All tibias were scanned with the same variables (pixel size, $18 \mu \mathrm{m} ; 50 \mathrm{kV} ; 0.5$-mm Al filter). The relative trabecular bone volume and other trabecular variables (number, thickness, and separation) were quantified with the SkyScan CtAn software version 1.13. For the bone phenotype of $O s m$ or $O s m r^{-1-}$ mice, the metaphyseal secondary spongiosa of the proximal tibia of 7-to 8-weekold littermates was analyzed. For bone defect experiments, the area of interest corresponded to the injured area.

\section{Real-Time PCR}

After sacrifice by $\mathrm{CO}_{2}$ inhalation, soft tissue of left hindlimbs was removed (soft or hard callus around bone defect was preserved). Total RNA was extracted from bone defectcontaining tissue samples of $0.5 \mathrm{~cm}$ long by using TRIzol reagent and DI25 Ultra-Turrax (IKA, Staufen, Germany) on ice and at the indicated time after surgery. First-strand cDNA was synthesized from $1 \mu \mathrm{g}$ of total RNA by using ThermoScript RT-PCR System (Invitrogen Life Technologies, CergyPontoise, France). The real-time PCR contained $10 \mathrm{ng}$ of reverse-transcribed total RNA, SYBR green buffer (Bio-Rad, Marnes-la-Coquette, France), and $300 \mathrm{nmol} / \mathrm{L}$ previously described primers. ${ }^{10,17,18}$ Quantitative PCRs were performed on a CFX96 Real-Time PCR Detection System (Bio-Rad). Analysis was performed with glyceraldehyde3-phosphate dehydrogenase or hypoxanthine-guanine phosphoribosyltransferase used as invariant controls and results were expressed as $2^{-\Delta \mathrm{Ct}}$.

\section{Histology and Immuohistochemistry}

Hindlimbs were fixed in $4 \%$ paraformaldehyde (SigmaAldrich, St Louis, MO) at room temperature and then
A

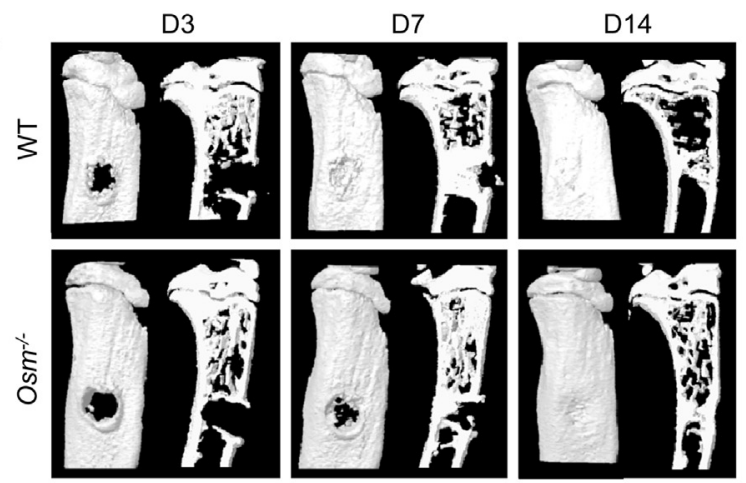

B
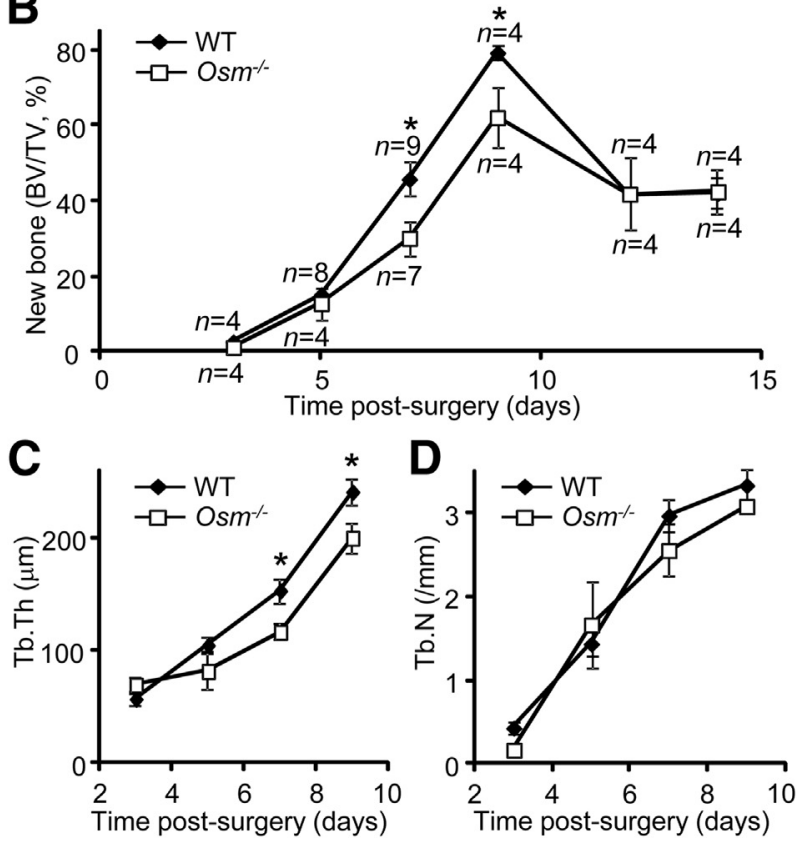

Figure 3 Delayed bone healing in $0 \mathrm{sm}^{-/-}$mice. A: At indicated days after injury (D3, D7, and D14), tibias from $0 \mathrm{sm}^{-/-}$and WT littermates were analyzed by X-ray microCT scan. Representative sagittal sections are shown. B: Quantification of newly formed bone within the injury site by microCT (BV/TV). C and D: Relative Tb.Th (C) and Tb.N (D) of the intramedullar woven bone. Data are expressed as means \pm SEM. $n=4$ to 9 mice in each group as indicated in B. ${ }^{*} P<0.05$ versus WT. BV/TV, trabecular bone volume; microCT, micro-computed tomography; Tb.N, trabecular number; Tb.Th, trabecular thickness; WT, wild-type.

decalcified for 96 hours in $4.13 \%$ EDTA (Sigma-Aldrich) and $0.2 \%$ paraformaldehyde in PBS at $\mathrm{pH} 7.4$ to 7.6 by using the KOS Microwave Histostation (Milestone, Kalamazoo, MI). Once decalcified, all samples were embedded in paraffin.

Sections $(4 \mu \mathrm{m}$ thick) were stained by Masson trichrome staining as described previously. ${ }^{19}$ Tartrate-resistant acid phosphatase (Trap) staining was performed by 1-hour incubation in 1 $\mathrm{mg} / \mathrm{mL}$ naphthyl phosphate and $1 \mathrm{mg} / \mathrm{mL}$ Fast Red Salt (SigmaAldrich) solution ( $\mathrm{pH}$ 5.2). The counterstain was done with Mayer hematoxylin. Immunostaining was performed as described previously ${ }^{10,18}$ with the following primary antibodies at the indicated concentration: rabbit anti-osterix (OSX; dilution 1:800; Abcam, Cambridge, MA), goat anti-ionized calciumbinding adapter molecule 1 (IBA1; dilution 1:1000; 
A
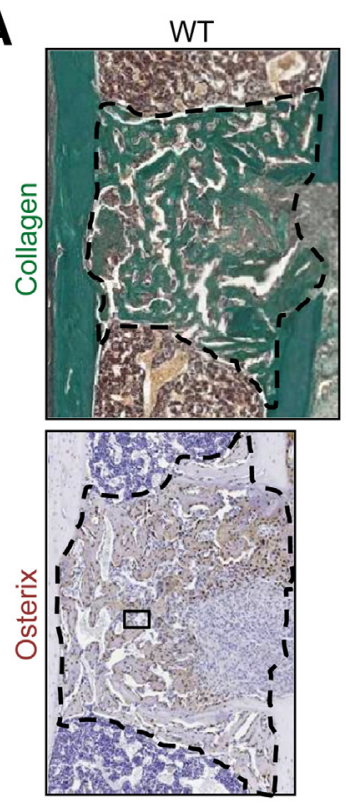

$\mathrm{Osm}^{-1-}$

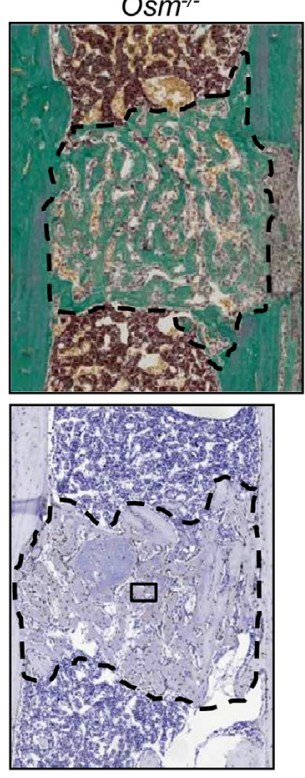

B
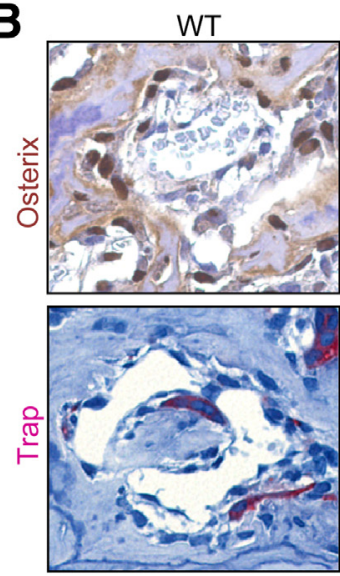
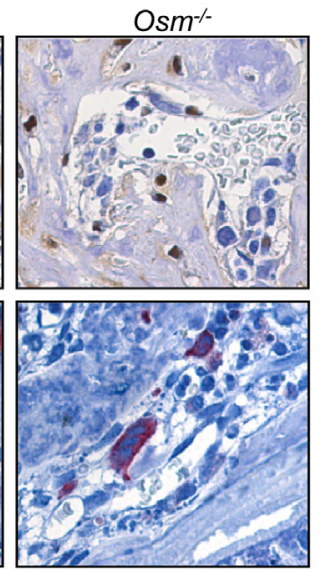

C
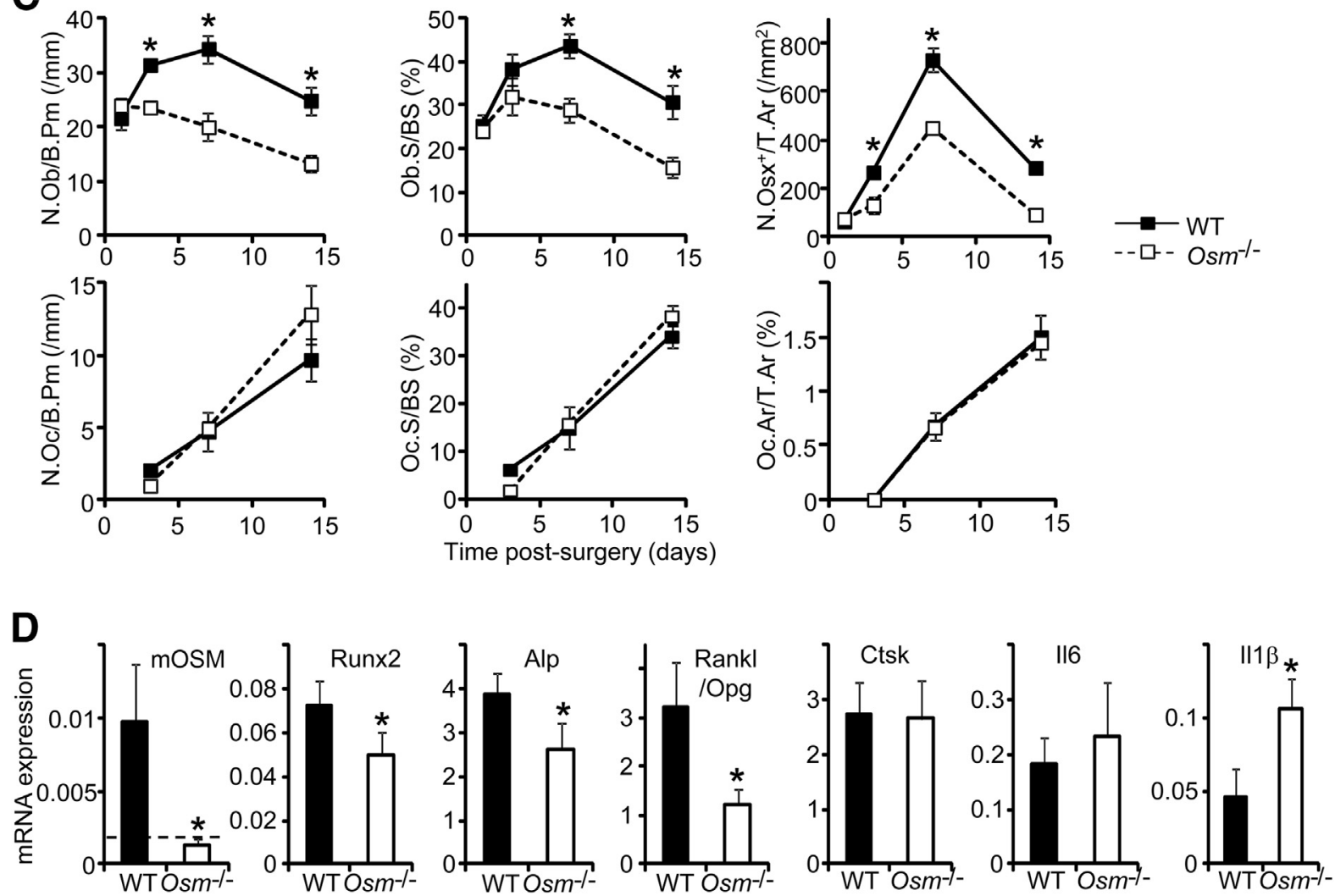

Figure $4 \mathrm{Osm}^{-1-}$ mice have reduced number of osteoblasts within the injury site. A and B: Seven days after injury animals were sacrificed, and tibial sections were stained with Masson trichrome (collagen fibers stained in green) or for osterix (osteoblastic cells stained in brown) and Trap (osteoclasts stained in red). Representative images are shown. Injury sites are indicated by dotted areas. The upper panels in B are enlarged images of the boxed areas in A. C: Histomorphometric values within the injury site were calculated at indicated time points after bone injury by using the NDPView2 software for bone formation parameters (N.Ob/B.Pm and Ob.S/BS) and for bone resorption parameters (N.Oc/B.Pm and Oc.S/BS). The N.Osx ${ }^{+} /$T.Ar and the Oc.Ar/T.Ar were quantified with ImageJ software. D: mRNA expression of indicated genes was analyzed 7 days after injury by real-time PCR. Hprt was used as an invariant control. The dotted line represents threshold under which Osm is considered not expressed. Data are expressed as means \pm SEM. $n=3$ mice in each group at each time point, except $n=4$ mice in the WT group at day 7 (C); $n=4$ mice in each group (D). ${ }^{*} P<0.05$ versus WT. Original magnification: $\times 5$ (A); $\times 40$ (B). Alp, alkaline phosphatase; Ctsk, cathepsin K; N.0b/B.Pm, number of osteoblasts/bone perimeter; N.0c/B.Pm, number of osteoclasts/bone perimeter; N.0sx ${ }^{+} /$T.ar, number of osterix ${ }^{+}$osteoblastic cells/tissue area; 0b.S/BS, osteoblast surface/bone surface; 0 c.Ar/T.Ar, osteoclast area/tissue area; $0 \mathrm{c} . \mathrm{S} / \mathrm{BS}$, osteoclast surface/bone surface; $0 \mathrm{pg}$, osteoprotegerin; m0SM, murine oncostatin M; Rankl, receptor activator of NF- $\kappa$ B ligand; Runx2, runt related transcription factor 2; Trap, tartrate-resistant acid phosphatase; WT, wild-type. 
Abcam), goat anti-mOSM (dilution 1:50; R\&D Systems, Minneapolis, MN), goat anti-Osmr (dilution 1:100; R\&D Systems), and rabbit anti-phospho-Stat3 (Tyr705; dilution 1:400; Cell Signaling Technologies, Beverly, MA). Histomorphometric values were calculated after Trap staining, within the secondary spongiosa or in selected area in bone defect, using the NDPView software version 2.3.1 (Hamamatsu, Massy, France) for bone formation parameters [number of osteoblasts/bone perimeter (N.Ob/B.Pm), osteoblast surface/bone surface (Ob.S/BS)] and for bone resorption parameters (N.Oc/B.Pm and Oc.S/BS) following the recommendations of the American Society for Bone and Mineral Research. ${ }^{20}$

The number of osterix + osteoblastic cells/tissue area (N.Osx $+/ \mathrm{T} . \mathrm{Ar}$ ) and the osteoclast area/tissue area (Oc.Ar/ T.Ar) were quantified with the ImageJ software version $1.43 \mathrm{u}$ (NIH, Bethesda, MD) as described. ${ }^{10}$ All analyses were assessed after scanning the slides with the NanoZoomer 2.0-RS system (Hamamatsu).

\section{Statistical Analysis}

Results were analyzed with Mann-Whitney or unpaired $t$-test by using GraphPad InStat software version 3.02 (GraphPad Inc., San Diego, CA). Results are given as means \pm SD or SEM as indicated, and $P<0.05$ was considered significant.

\section{Results}

\section{OSM Is Expressed in the Bone Injury Site}

We used a tibia injury model in C57BL/6 mice in which bone heals mainly through intramembranous ossification. ${ }^{4}$ In this model, the standard phases of stabilized fracture healing were observed, that is, an initial inflammatory phase within the first 3 days, a bone modeling phase with intramedullar woven bone deposition that culminates around day 7 , and later a bone remodeling phase in which osteoclasts largely resorbed the woven bone (Supplemental Figure S1). Rarely, cartilaginous tissues were observed in periosteal area but never in the medullar cavity (not shown). Interestingly, mOSM mRNA amount was induced during the initial inflammatory phase ( 8 hours after injury), together with Il- 6 and Il-1 $\beta$ but not tumor necrosis factor $\alpha$ (Figure 1). Expression of the antiinflammatory cytokine IL-10 peaked later at day 3, whereas typical osteoblast and bone formation markers (runt-related transcription factor 2, Osx, alkaline phosphatase, and bone sialoprotein) arose at approximately days 5 to 7 . Osteoclastrelated markers [receptor activator of NF- $\kappa \mathrm{B}$ ligand (Rankl), cathepsin $\mathrm{K}$, and Trap] were highly induced at day 10 after injury (Figure 1). Expression of osteoprotegerin (Opg), a Rankl decoy receptor, followed the expression profile of bone formation markers; the Rankl/Opg ratio being thus strongly induced after day 10 (Figure 1).

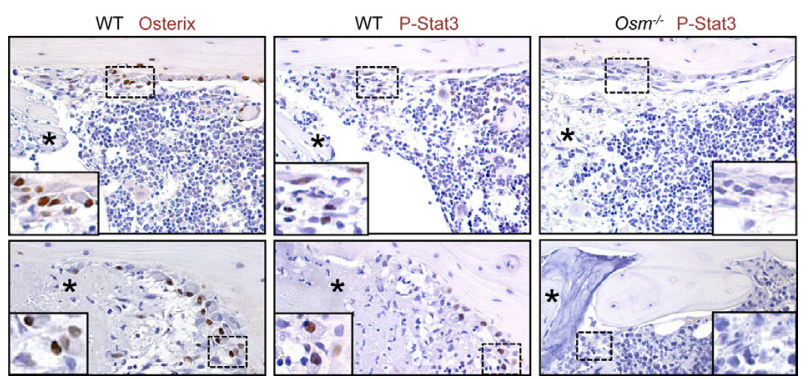

Figure 5 Activation of Stat3 is reduced in $0 \mathrm{sm}^{-/-}$mice. Activation of Stat3 was analyzed 1 day after injury by immunohistochemistry by using an anti-P-Stat3 antibody. Representative images from two different animals are shown. A serial section is shown for 0sterix staining. The insets (boxed areas) are enlarged images of dotted boxes. Asterisks indicate hematoma with eventual bone debris. $n=3$ mice in each group. Original magnification: $\times 10$ (main images); $\times 40$ (insets). P-Stat3, phospho-Stat3; WT, wild-type.

\section{OSM Expression Is Repressed by Macrophage Depletion}

Efficient macrophage depletion during 7 to 8 days or more is known to repress physiologic bone formation, PTH bone anabolic actions, ${ }^{21,22}$ or bone reconstruction by osteoblasts in this tibia injury model, ${ }^{4}$ and we next wondered whether it would also repress mOSM expression. As revealed by immunohistochemistry with the pan-macrophage marker IBA1, macrophages were located throughout the bone marrow and eventually in contact with the endoste (Figure 2, A, C, and E). Three days after treatment with the macrophage-depleting agent clodronate liposome largely reduced the number of $\mathrm{IBA} 1^{+}$ macrophages, whereas osteoblasts or osteocytes were not altered (Figure 2, A, C, and E and Supplemental Figure S2). Eight hours after bone injury, IBA $1^{+}$macrophages were numerous at the periphery of the hematoma and again were efficiently depleted with clodronate liposomes (Figure 2, A, C, and $\mathrm{E})$. At this location of the tibia (diaphysis) and early time point after bone injury (8 hours), osteoclasts were rare in PBS or clodronate liposome-treated mice (not shown) (Supplemental Figure S1). Therefore, this short-term treatment with clodronate liposome resulted in a specific depletion of macrophages in the bone marrow and in the bone injury site, osteoblasts and osteocytes being unaltered, and osteoclasts being almost absent.

By immunohistochemistry, mOSM protein was found in the cytoplasm of cells throughout the bone marrow and in the periphery of the hematoma (Figure 2, $\mathrm{B}, \mathrm{D}$, and F). $\mathrm{mOSM}^{+}$cells had a structure compatible with IBA $1^{+}$macrophages and were also drastically depleted with clodronate liposomes (Figure 2, B, $\mathrm{D}$, and F). As described previously,$^{13}$ osteoblasts and osteocytes were also found to express mOSM, but they were not modified after this short treatment with clodronate liposome (Figure 2F). Depletion of macrophages repressed the early induction of mOSM mRNA expression 8 hours after bone injury, and similar results were also observed for Il-6 or Il-1b expression (Figure 2G). Together, these results strongly suggested that soon after bone injury macrophages were major cell sources of these inflammatory cytokines. Note that clodronate liposomes did not ablate mOSM expression in the absence of bone injury (Figure $2 \mathrm{G}$ ), suggesting that other cells such as osteoblasts or 
osteocytes could be implicated in the basal expression of this cytokine.

\section{Osm Deficiency Leads to Delayed Bone Reconstruction}

To study the potent role of mOSM in bone healing, we next used Osm-deficient mice. On the basis of microCT, quantitative bone histomorphometry, and immunohistochemistry, $\mathrm{Osm}^{-1-}$ mice appeared to have a grossly normal bone phenotype by 7 to 8 weeks of age (Supplemental Figure S3). Compared with WT littermates, trabecular bone volume, thickness, and number and osteoblast or osteoclast number and surface were identical, and only a slight decrease in the trabecular separation was noticed. However, 7 to 9 days after tibial injury, we observed a significant reduction in the amount of new intramedullar bone formed at the injured site for $\mathrm{Osm}^{-1-}$ mice by microCT (Figure 3). By day 12 to 14 after injury, corresponding to the remodeling phase, the intramedullar bone was reduced and the cortical bone was formed in both WT and $\mathrm{Osm}^{-/-}$mice, and, overall, no difference was found anymore in bone volumes between the two genotypes (Figure 3, A and B). Reduced new intramedullar bone in $\mathrm{Osm}^{-1-}$ mice at day 7 to 9 was because of reduced thickness of the newly formed bony trabeculae, trabecular number being not statistically different (Figure 3, C and D).

Masson trichrome staining revealed a reduced amount of collagen deposition in the injured tibia of $\mathrm{Osm}^{-/-}$mice at day 7 (Figure 4A). The intramedullar new woven bone appeared as an irregular network of bony trabeculae, and it was reduced and less dense in $\mathrm{Osm}^{-1-}$ mice. As early as day 3 until day 14, there was also a significant reduction in osteoblast number (N.Ob/B.Pm) and surface (Ob.S/BS), and in the number of $\mathrm{Osx}^{+}$osteoblastic cells (N.Osx ${ }^{+} / \mathrm{T}$.Ar) in $\mathrm{Osm}^{-/-}$mice (Figure 4, A-C). Osx ${ }^{+}$ cells were mainly osteoblasts lining the bony trabeculae, but preosteoblasts (not in contact with bone) or osteocytes (embedded into bone matrix) were eventually also positive for Osx staining (Figure 4B and Supplemental Figure S1). In contrast, Trap ${ }^{+}$ osteoclast number (N.Oc/B.Pm), surface (Oc.S/BS), or area (Oc.Ar/T.Ar) was normal throughout the healing period in $\mathrm{Osm}^{-/-}$mice (Figure 4, B and C). At day 7, mRNA amounts of runt-related transcription factor 2, alkaline phosphatase, and the Rankl/Opg ratio were all reduced, and cathepsin $\mathrm{K}$ and Il-6 amounts were normal, whereas Il-1 $\beta$ amount was elevated in $\mathrm{Osm}^{-1-}$ mice (Figure 4D). Together, these results indicated that Osm deficiency led to a transient delay only in the initial modeling phase of the bone healing process, with reduced woven bone apposition by osteoblasts. In contrast, bone remodeling and osteoclasts were not affected despite altered expression of inflammatory/osteoclastogenic cytokines (Rankl/ Opg and Il-1 $\beta$ ).

\section{Stat3 Is Activated during the Initial Inflammatory Phase}

STAT3 is the main transcription factor activated by OSM and is necessary for this cytokine to induce osteogenesis in
MSCs. ${ }^{10}$ One day after bone injury, Stat3 was found phosphorylated and thus activated in a discrete population of endosteal cells located at the hedge of the hematoma (Figure 5). On serial sections, these cells stained positive for Osx and were therefore of the osteoblastic lineage (Figure 5 and Supplemental Figure S1). Interestingly, we observed reduced activation of Stat3 in $\mathrm{Osm}^{-/-}$mice (Figure 5), indicating that mOSM was implicated early in activation of Stat3 in endosteal osteoblastic cells. At later time points, Stat 3 activation was low in WT or $O \mathrm{sm}^{-1-}$ mice, especially in mature osteoblasts in contact with the newly formed woven bone (data not shown).

\section{Osmr Deficiency Leads to Reduced Bone Healing}

In human MSCs, OSM recruits a receptor composed of Gp130 and OSMR to activate STAT3 and osteogenesis. ${ }^{10}$ Murine osteoblasts also express the Osmr, ${ }^{13}$ and we determined that macrophage depletion with clodronate liposome did not alter Osmr expression in osteoblasts (Supplemental Figure S2). As observed with $\mathrm{Osm}^{-1-}$ mice, 7- to 8-weekold $\mathrm{Osmr}^{-/-}$mice had normal bone architecture, osteoblasts, and osteoclasts (Supplemental Figure S3). However, 7 days after tibia injury, they had reduced woven bone formation (Figure 6A), osteoblast number and surface, and reduced number of $\mathrm{Osx}^{+}$osteoblastic cells within the injury site (Figure 6, B-D), a result similar to the one observed with $\mathrm{Osm}^{-1-}$ mice (Figures 3 and 4). Unexpectedly, Osmr ${ }^{-1-}$ mice also had reduced osteoclast number, surface, and area compared with WT littermates in this bone injury model (Figure 6, C and D), in contrast to $\mathrm{Osm}^{-1-}$ mice which had normal osteoclasts (Figure 4).

\section{Discussion}

The innate immune system is activated within a few hours after bone injury, with platelets, neutrophils, and macrophages being the first cells present in the inflamed fractured site. At that time, a wide variety of inflammatory cytokines, growth factors, and chemokines are released to further fuel leukocyte recruitment and to start bone healing through activation of mesenchymal progenitor cells. With the use of the mouse model, we show that mOSM is part of this early anabolic cytokine cocktail with an important role in the initial phase of bone apposition, that is, bone modeling, whereas the subsequent remodeling phase does not implicate this cytokine. Preliminary results also indicated that OSM is highly expressed in human fractured bones (data not shown). Although additional experiments are compulsory, it is tempting to speculate that OSM also has a role in human bone healing.

\section{Macrophages and Bone Healing}

Macrophages are detected early during human or mouse fracture healing, and their depletion by using clodronate 

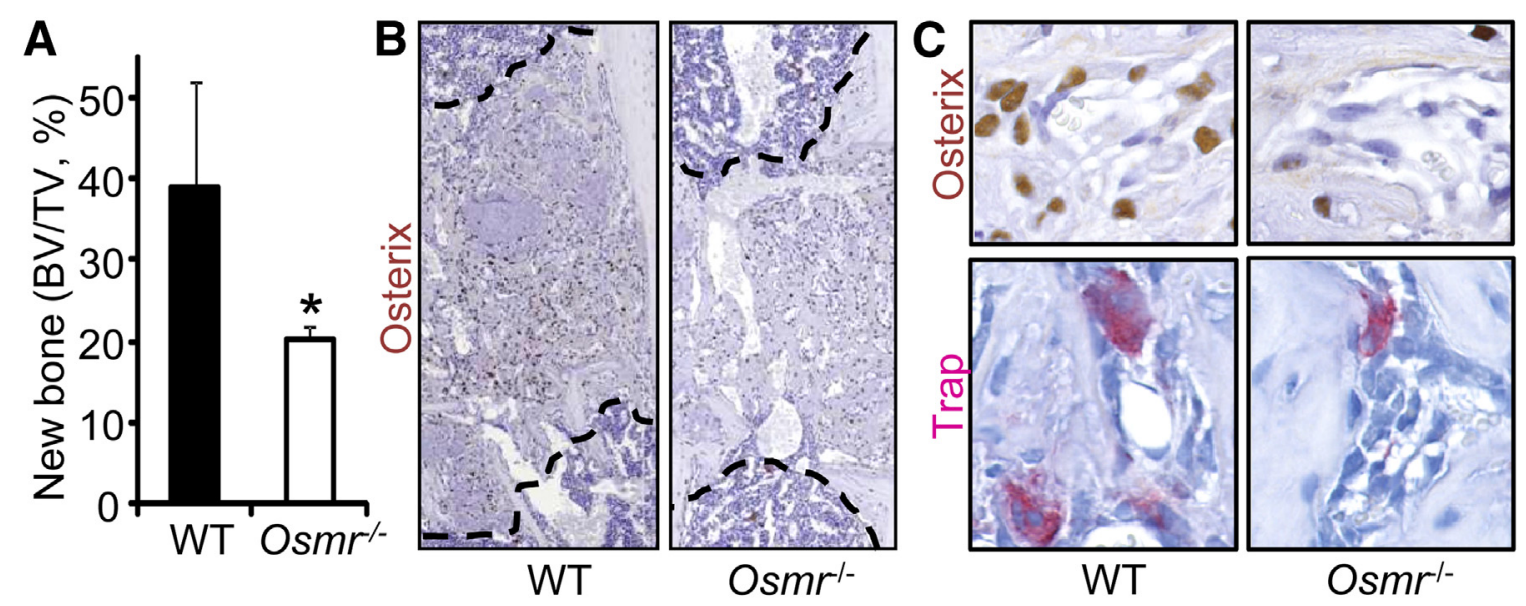

D
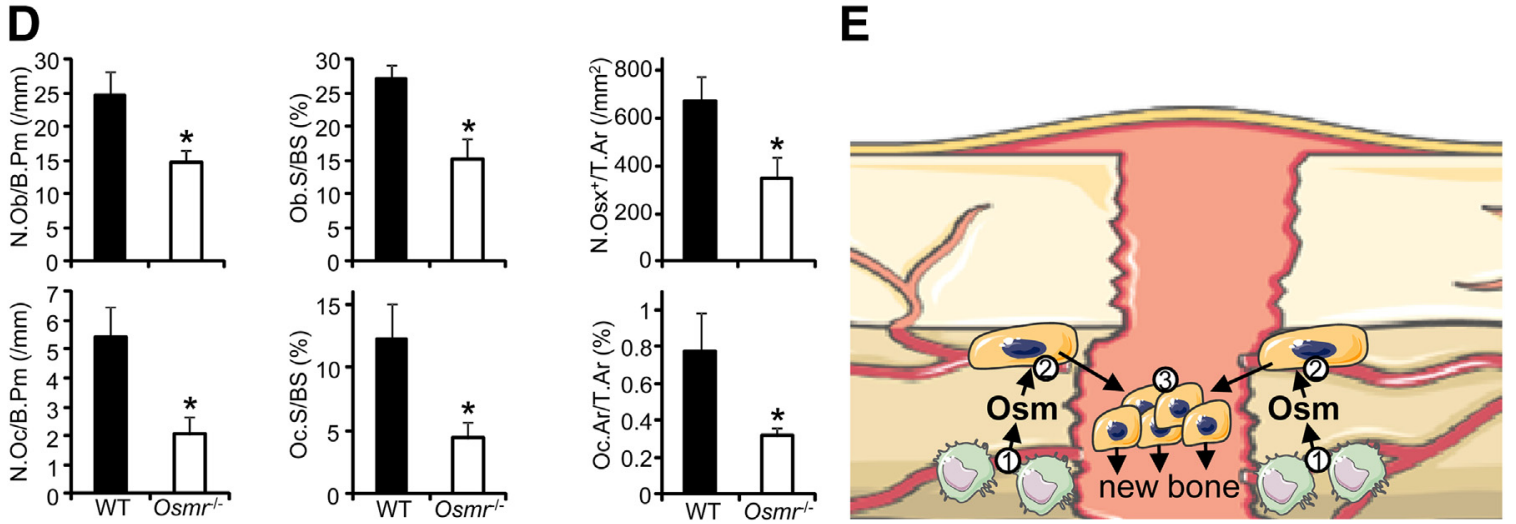

Figure $6 \mathrm{Osmr}^{-1-}$ mice have reduced bone healing. A: Seven days after injury, quantification of newly formed bone within the injury site was realized by microcomputed tomography (relative bone volume). B and C: Seven days after injury, animals were sacrificed and analyzed for osterix (osteoblastic cells stained in brown) and Trap (osteoclasts stained in red) staining. Representative images are presented. Injury sites are indicated by dotted areas. D: Histomorphometric values within the injury sites were calculated with the NDPView2 software for bone formation parameters (N.Ob/B.Pm and Ob.S/BS) and for bone resorption parameters (N.0c/B.Pm and Oc.S/BS). The N.Osx $/$ T.Ar and the 0c.Ar/T.Ar were quantified with ImageJ software. E: Proposed model for the role of m0SM during bone healing. Eight hours after bone injury, mOSM is secreted by macrophages (1). At 24 hours, the 0 smr-Stat3 or other pathways are activated in endosteal osteoblastic cells (2). At 3 to 7 days, this leads to sustained proliferation, migration, and/or differentiation of osteoblasts, and thus enhanced intramembranous bone formation (3). Data are expressed as means \pm SEM. $n=4$ WT mice (A); $n=30 \mathrm{smr}^{-/-}$mice (A); $n=4$ mice in each group (D). ${ }^{*} P<0.05$ versus WT. Original magnification: $\times 5$ (B); $\times 40$ (C). BV/TV, trabecular bone volume; N.0b/B.Pm, number of osteoblasts/bone perimeter; N.0c/B.Pm, number of osteoclasts/bone perimeter; N.0xs ${ }^{+} / \mathrm{T}_{\text {.Ar, number of osterix }}^{+}$ osteoblastic cells/tissue area; 0b.S/BS, osteoblast surface/bone surface; 0c.Ar/T.ar, osteoclast area/tissue area; 0c.S/BS, osteoclast surface/bone surface; m0SM, murine oncostatin $\mathrm{M}$; Trap, tartrate-resistant acid phosphatase.

liposome was shown to suppress bone deposition during bone healing. ${ }^{4,23}$ Until recently, however, the identity of the macrophage-secreted factor(s) that drive(s) bone reconstruction remained elusive. With the use of in vitro human primary cultures, we previously showed that the main bone anabolic factor produced by monocytes/macrophages is OSM. ${ }^{10}$ Here, with the use of a mouse tibia injury model, a macrophage-depletion strategy with clodronate liposome and $O s m$ knockout mice, we propose that early after bone injury $\mathrm{IBA}^{+}$macrophages efficiently produce mOSM to promote bone repair (Figure 6E). However, this assumption needs to be confirmed, for example, by using mice with specific Osm deficiency in macrophages. Indeed, other cell types such as osteoblasts, osteocytes, T lymphocytes, or neutrophils are known to produce this cytokine, ${ }^{13,24,25}$ but after bone injury the main cell sources of mOSM appeared to be macrophages that are highly sensitive to clodronate liposome.

\section{Anabolic Pathways for Bone Formation}

OSM is not the sole anabolic agent implicated in bone healing and should act together with other important soluble mediators. Previous studies demonstrated that OSM acts in synergy with bone morphogenetic protein 2 to induce osteogenesis in human MSCs. ${ }^{12}$ Through inhibition of sclerostin expression in osteocytes, mOSM could also release the Wnt signaling pathways to induce bone formation in vivo. ${ }^{13}$ Moreover, Osmr expression is induced by PTH and the anabolic effect of PTH is reduced in $\mathrm{Omsr}^{-1-}$ mice, suggesting important crosstalk between the OSM and PTH signaling pathways. ${ }^{26}$ It should be noted that tumor necrosis factor $\alpha$ and OSM, only when applied together, increased alkaline phosphatase activities and in vitro calcification by vascular smooth muscle cells. ${ }^{27}$ Future studies should certainly unravel how these bone anabolic agents act in network to sustain bone healing. 
We and others previously described that two other IL-6-type cytokines have a role in induction of osteogenesis: IL-6 itself and leukemia inhibitory factor (LIF). ${ }^{10,28}$ All these cytokines activate the common Gp130 receptor chain and STAT3, and they most presumably induce additive or redundant signals during bone healing. Indeed, Gp130-deficient mice or mice with osteoblast-specific disruption of the Gp130 (Il6st) or Stat3 gene have much more severe bone phenotype than $\mathrm{Osm}$ or $\mathrm{Osmr}^{-1-}$ mice, with reduced bone formation and osteopenia. ${ }^{29-32}$ Moreover, STAT3 mutations cause a rare human immunodeficiency disease called hyper-IgE syndrome that presents reduced bone mineral density and recurrent pathologic fractures. ${ }^{33} \mathrm{We}$ show here that young adult $\mathrm{Osm}$ or $\mathrm{Osmr}^{-1-}$ mice do not have an abnormal bone phenotype, but after bone injury these mice have reduced bone healing, with reduced number of osteoblastic cells and reduced early activation of Stat3 in osteoblastic endosteal cells. These results suggest that mOSM does not have a significant role during bone development or physiologic bone remodeling in young adult mice, but after bone injury this cytokine is highly expressed and has an important role in the activation of mesenchymal progenitor cells.

Other studies described that $\mathrm{Osmr}^{-/-}$mice are osteopetrotic with reduction in osteoblasts and osteoclasts. ${ }^{13}$ This discrepancy with our results could be explained by differences in animal housing and breeding, but these protocols are rarely published and thus difficult to compare. In addition, $O s m r^{-1-}$ neonates were already shown to have normal osteoblasts or osteoclasts (as in the present study that used 7- to 8-week-old mice), and only 12-week-old $\mathrm{Osmr}^{-1-}$ mice had altered bone cells, ${ }^{13}$ suggesting that reduced bone remodeling is associated with aging in $\mathrm{Osmr}^{-1-}$ mice. Importantly, $\mathrm{Osmr}^{-1-}$ mice also develop adipose tissue inflammation, insulin resistance, and obesity by 16 to 35 weeks of age, ${ }^{34}$ conditions that can have important confounding effects on bone cells. Similarly Il6-deficient mice, despite having a normal bone phenotype, develop osteopenia with aging and have delayed callus mineralization in the early stages of fracture healing. ${ }^{7,35}$ More recently, it was shown that mice with osteoblast-specific disruption of Gp130 do not have osteopenia until 12 weeks of age. ${ }^{32}$

\section{Intramenbranous versus Endochondral Ossification during Bone Healing}

Bone fractures can heal through different modes of ossification. In the bone injury model used in the present study, intramembranous bone apposition is mainly involved with the bone matrix being directly produced by osteoblasts. $\mathrm{Osm}$ deficiency impairs the early steps of intramembranous bone formation in this model, presumably by acting directly on the recruitment, proliferation, and/or osteoblast differentiation of mesenchymal progenitor cells initially located at the endosteal surface near the fractured inflamed site (Figure 6E). Indeed, early activation of Stat3, expression of several osteoblast markers, and collagen deposition are reduced in the absence of mOSM. However, mOSM does not have a role later for cortical bone reconstruction, at a time when its expression is largely normalized. In culture, OSM can induce proliferation, osteogenesis, and bone matrix formation by purified MSCs. ${ }^{10,36}$ Injection of mOSM also induces an increase of mouse calvarial thickness, a model of intramembranous ossification, ${ }^{13}$ and OSM can enhance bony filling in a rabbit critical calvarial defect model ${ }^{37}$ In contrast to calvarial bones, long bones develop and can heal through both intramembranous and/or endochondral ossification, the later being predominant for large critical bone defects. We previously described that OSM could induce the terminal stage of chondrogenesis to the hypertrophic chondrocyte, which is a necessary step for endochondral bone formation especially at the growth plate. ${ }^{38}$ In addition, mOSM overexpression induces abnormal cartilaginous growth (chondrophyte) formation and growth plate damage. ${ }^{39}$ In Osm or $O s m r^{-1-}$ mice, we did not observe alteration at the growth plate, long bone sizes were normal, and the amount of cartilaginous matrix formed after bone injury was comparable with WT littermates, although periosteal endochondral ossification was only rarely observed in this bone injury model (data not shown). Whether mOSM has an important role in consolidation of larger critical long bone defects through sustained intramembranous and/or endochondral ossification thus deserves further investigations.

\section{mOSM and Bone Resorption}

The last step of bone healing relies on bone remodeling. At this stage, osteoclasts resorb the woven bone, which is later replaced by more mature lamellar bone especially cortical bone. mOSM is able to induce osteoclast formation through increased Rankl expression in osteoblastic or chondroblastic cells. ${ }^{13,38}$ With the use of $\mathrm{Osmr}^{-1-}$ mice and a Lif receptor (Lifr) antagonist, it was suggested that mOSM, when acting through the Osmr, induces Rankl, bone resorption, and bone formation, whereas mOSM, when acting through the Lifr, reduces sclerostin expression in osteocytes and thus induces bone formation independently of bone resorption. ${ }^{13}$ In addition, Lif- or Lifr-deficient mice have a striking increased number of giant osteoclasts, ${ }^{40,41}$ suggesting that mOSM sustains bone resorption through the Osmr but restrains it through the Lifr. Another cytokine, Il-31, can also recruit and activate the Osmr, but it did not appear to have a role on bone cells. ${ }^{10,13}$ After bone injury, we observed that $\mathrm{Osmr}^{-/-}$ mice had reduced osteoclasts, but $\mathrm{Osm}^{-1-}$ mice, despite having a reduced Rankl/Opg ratio, had no alteration of osteoclasts or cathepsin $\mathrm{K}$ expression. These results would suggest that an additional receptor for mOSM, maybe the Lifr, effectively exists in mice and counteracts the Osmr effect on osteoclast formation. Another possibility, not excluding the first one, is that compensatory mechanisms maintain osteoclast formation in $\mathrm{Osm}^{-1-}$ mice, such as induced expression of Il-1 $\beta$ (Figure 4D) which is a known inflammatory cytokine that directly stimulates osteoclastogenesis and survival of osteoclast precursors. ${ }^{42}$ 
Together these data suggest that mOSM, when signaling through the Osmr-Stat3 or additional pathways, is an important factor in the first stages of bone healing (Figure 6E). This inflammatory cytokine appears unique in that it induces intramembranous bone formation but leaves bone resorption largely unaltered. mOSM could therefore represent an interesting new anabolic treatment for unconsolidated bone fractures.

\section{Acknowledgments}

We thank Prof. Jean-Claude Lecron (University of Poitiers, France) and Dr. Hans Yssel (INSERM U945, Paris, France) for providing Osm- and Osmr-deficient mice, Prof. François Gouin (INSERM U957, Nantes, France) for human bone samples, and Dr. Frédéric Lezot (INSERM U957, Nantes, France) for helpful advice on mouse breeding.

\section{Supplemental Data}

Supplemental material for this article can be found at http://dx.doi.org/10.1016/j.ajpath.2014.11.008.

\section{References}

1. Glass GE, Chan JK, Freidin A, Feldmann M, Horwood NJ, Nanchahal J: TNF-alpha promotes fracture repair by augmenting the recruitment and differentiation of muscle-derived stromal cells. Proc Natl Acad Sci U S A 2011, 108:1585-1590

2. Rachner TD, Khosla S, Hofbauer LC: Osteoporosis: now and the future. Lancet 2011, 377:1276-1287

3. Rozen N, Lewinson D, Bick T, Jacob ZC, Stein H, Soudry M: Fracture repair: modulation of fracture-callus and mechanical properties by sequential application of IL-6 following PTH 1-34 or PTH 28-48. Bone 2007, 41:437-445

4. Alexander KA, Chang MK, Maylin ER, Kohler T, Muller R, Wu AC, Van Rooijen N, Sweet MJ, Hume DA, Raggatt LJ, Pettit AR: Osteal macrophages promote in vivo intramembranous bone healing in a mouse tibial injury model. J Bone Miner Res 2011, 26:1517-1532

5. Schindeler A, McDonald MM, Bokko P, Little DG: Bone remodeling during fracture repair: the cellular picture. Semin Cell Dev Biol 2008, 19:459-466

6. Xie C, Ming X, Wang Q, Schwarz EM, Guldberg RE, O'Keefe RJ, Zhang X: COX-2 from the injury milieu is critical for the initiation of periosteal progenitor cell mediated bone healing. Bone 2008, 43: $1075-1083$

7. Yang X, Ricciardi BF, Hernandez-Soria A, Shi Y, Pleshko Camacho N, Bostrom MP: Callus mineralization and maturation are delayed during fracture healing in interleukin-6 knockout mice. Bone 2007, 41:928-936

8. Toben D, Schroeder I, El Khassawna T, Mehta M, Hoffmann JE, Frisch JT, Schell H, Lienau J, Serra A, Radbruch A, Duda GN: Fracture healing is accelerated in the absence of the adaptive immune system. J Bone Miner Res 2011, 26:113-124

9. Danks L, Takayanagi H: Immunology and bone. J Biochem 2013, 154: 29-39

10. Guihard P, Danger Y, Brounais B, David E, Brion R, Delecrin J, Richards CD, Chevalier S, Redini F, Heymann D, Gascan H, Blanchard F: Induction of osteogenesis in mesenchymal stem cells by activated monocytes/macrophages depends on oncostatin M signaling. Stem Cells 2012, 30:762-772
11. Nicolaidou V, Wong MM, Redpath AN, Ersek A, Baban DF, Williams LM, Cope AP, Horwood NJ: Monocytes induce STAT3 activation in human mesenchymal stem cells to promote osteoblast formation. PLoS One 2012, 7:e39871

12. Fernandes TJ, Hodge JM, Singh PP, Eeles DG, Collier FM, Holten I, Ebeling PR, Nicholson GC, Quinn JM: Cord blood-derived macrophage-lineage cells rapidly stimulate osteoblastic maturation in mesenchymal stem cells in a glycoprotein-130 dependent manner. PLoS One 2013, 8:e73266

13. Walker EC, McGregor NE, Poulton IJ, Solano M, Pompolo S, Fernandes TJ, Constable MJ, Nicholson GC, Zhang JG, Nicola NA, Gillespie MT, Martin TJ, Sims NA: Oncostatin M promotes bone formation independently of resorption when signaling through leukemia inhibitory factor receptor in mice. J Clin Invest 2010, 120: $582-592$

14. Morikawa Y, Tamura S, Minehata K, Donovan PJ, Miyajima A, Senba E: Essential function of oncostatin $\mathrm{m}$ in nociceptive neurons of dorsal root ganglia. J Neurosci 2004, 24:1941-1947

15. Tanaka M, Hirabayashi $\mathrm{Y}$, Sekiguchi $\mathrm{T}$, Inoue $\mathrm{T}$, Katsuki $\mathrm{M}$, Miyajima A: Targeted disruption of oncostatin M receptor results in altered hematopoiesis. Blood 2003, 102:3154-3162

16. van Rooijen N: Liposomes for targeting of antigens and drugs: immunoadjuvant activity and liposome-mediated depletion of macrophages. J Drug Target 2008, 16:529-534

17. Duplomb L, Baud'huin M, Charrier C, Berreur M, Trichet V, Blanchard F, Heymann D: Interleukin-6 inhibits receptor activator of nuclear factor kappaB ligand-induced osteoclastogenesis by diverting cells into the macrophage lineage: key role of Serine727 phosphorylation of signal transducer and activator of transcription 3. Endocrinology 2008, 149:3688-3697

18. David E, Tirode F, Baud'huin M, Guihard P, Laud K, Delattre O, Heymann MF, Heymann D, Redini F, Blanchard F: Oncostatin M is a growth factor for Ewing sarcoma. Am J Pathol 2012, 181:1782-1795

19. Brounais B, Chipoy C, Mori K, Charrier C, Battaglia S, Pilet P, Richards CD, Heymann D, Redini F, Blanchard F: Oncostatin M induces bone loss and sensitizes rat osteosarcoma to the antitumor effect of Midostaurin in vivo. Clin Cancer Res 2008, 14:5400-5409

20. Dempster DW, Compston JE, Drezner MK, Glorieux FH, Kanis JA, Malluche H, Meunier PJ, Ott SM, Recker RR, Parfitt AM: Standardized nomenclature, symbols, and units for bone histomorphometry: a 2012 update of the report of the ASBMR Histomorphometry Nomenclature Committee. J Bone Miner Res 2013, 28:2-17

21. Chang MK, Raggatt LJ, Alexander KA, Kuliwaba JS, Fazzalari NL, Schroder K, Maylin ER, Ripoll VM, Hume DA, Pettit AR: Osteal tissue macrophages are intercalated throughout human and mouse bone lining tissues and regulate osteoblast function in vitro and in vivo. $\mathrm{J}$ Immunol 2008, 181:1232-1244

22. Cho SW, Soki FN, Koh AJ, Eber MR, Entezami P, Park SI, van Rooijen N, McCauley LK: Osteal macrophages support physiologic skeletal remodeling and anabolic actions of parathyroid hormone in bone. Proc Natl Acad Sci U S A 2014, 111:1545-1550

23. Andrew JG, Andrew SM, Freemont AJ, Marsh DR: Inflammatory cells in normal human fracture healing. Acta Orthop Scand 1994, 65:462-466

24. Lacreusette A, Lartigue A, Nguyen JM, Barbieux I, Pandolfino MC, Paris F, Khammari A, Dreno B, Jacques Y, Blanchard F, Godard A: Relationship between responsiveness of cancer cells to Oncostatin M and/or IL-6 and survival of stage III melanoma patients treated with tumour-infiltrating lymphocytes. J Pathol 2008, 216:451-459

25. Grenier A, Dehoux M, Boutten A, Arce-Vicioso M, Durand G, Gougerot-Pocidalo MA, Chollet-Martin S: Oncostatin M production and regulation by human polymorphonuclear neutrophils. Blood 1999, 93: 1413-1421

26. Walker EC, Poulton IJ, McGregor NE, Ho PW, Allan EH, Quach JM, Martin TJ, Sims NA: Sustained RANKL response to parathyroid hormone in oncostatin $\mathrm{M}$ receptor-deficient osteoblasts converts anabolic treatment to a catabolic effect in vivo. J Bone Miner Res 2012, 27:902-912 
27. Shioi A, Katagi M, Okuno Y, Mori K, Jono S, Koyama H, Nishizawa Y: Induction of bone-type alkaline phosphatase in human vascular smooth muscle cells: roles of tumor necrosis factor-alpha and oncostatin M derived from macrophages. Circ Res 2002, 91:9-16

28. Blanchard F, Duplomb L, Baud'huin M, Brounais B: The dual role of IL-6-type cytokines on bone remodeling and bone tumors. Cytokine Growth Factor Rev 2009, 20:19-28

29. Sims NA, Jenkins BJ, Quinn JM, Nakamura A, Glatt M, Gillespie MT, Ernst M, Martin TJ: Glycoprotein 130 regulates bone turnover and bone size by distinct downstream signaling pathways. J Clin Invest 2004, 113:379-389

30. Sims NA: gp130 Signaling in bone cell biology: multiple roles revealed by analysis of genetically altered mice. Mol Cell Endocrinol 2009, 310:30-39

31. Itoh S, Udagawa N, Takahashi N, Yoshitake F, Narita H, Ebisu S, Ishihara K: A critical role for interleukin-6 family-mediated Stat3 activation in osteoblast differentiation and bone formation. Bone 2006, 39:505-512

32. Johnson RW, Brennan HJ, Vrahnas C, Poulton IJ, McGregor NE, Standal T, Walker EC, Koh TT, Nguyen H, Walsh NC, Forwood MR, Martin TJ, Sims NA: The primary function of gp130 signaling in osteoblasts is to maintain bone formation and strength, rather than promote osteoclast formation. J Bone Miner Res 2014, 29:1492-1505

33. Holland SM, DeLeo FR, Elloumi HZ, Hsu AP, Uzel G, Brodsky N, Freeman AF, Demidowich A, Davis J, Turner ML, Anderson VL, Darnell DN, Welch PA, Kuhns DB, Frucht DM, Malech HL, Gallin JI, Kobayashi SD, Whitney AR, Voyich JM, Musser JM, Woellner C, Schaffer AA, Puck JM, Grimbacher B: STAT3 mutations in the hyperIgE syndrome. N Engl J Med 2007, 357:1608-1619

34. Komori T, Tanaka M, Senba E, Miyajima A, Morikawa Y: Lack of oncostatin $\mathrm{M}$ receptor beta leads to adipose tissue inflammation and insulin resistance by switching macrophage phenotype. J Biol Chem 2013, 288:21861-21875

35. de Hooge AS, van de Loo FA, Bennink MB, Arntz OJ, de Hooge P, van den Berg WB: Male IL-6 gene knock out mice developed more advanced osteoarthritis upon aging. Osteoarthritis Cartilage 2005, 13: $66-73$

36. Song HY, Jeon ES, Jung JS, Kim JH: Oncostatin M induces proliferation of human adipose tissue-derived mesenchymal stem cells. Int J Biochem Cell Biol 2005, 37:2357-2365

37. Moxham JP: Oncostatin-M enhances osteoinduction in a rabbit critical calvarial defect model. Laryngoscope 2007, 117:1790-1797

38. David E, Guihard P, Brounais B, Riet A, Charrier C, Battaglia S, Gouin F, Ponsolle S, Bot RL, Richards CD, Heymann D, Redini F, Blanchard F: Direct anti-cancer effect of oncostatin $M$ on chondrosarcoma. Int J Cancer 2011, 128:1822-1835

39. de Hooge AS, van de Loo FA, Bennink MB, Arntz OJ, Fiselier TJ, Franssen MJ, Joosten LA, Van Lent PL, Richards CD, van den Berg WB: Growth plate damage, a feature of juvenile idiopathic arthritis, can be induced by adenoviral gene transfer of oncostatin M: a comparative study in gene-deficient mice. Arthritis Rheum 2003, 48: $1750-1761$

40. Ware CB, Horowitz MC, Renshaw BR, Hunt JS, Liggitt D, Koblar SA, Gliniak BC, McKenna HJ, Papayannopoulou T, Thoma B, et al: Targeted disruption of the low-affinity leukemia inhibitory factor receptor gene causes placental, skeletal, neural and metabolic defects and results in perinatal death. Development 1995, 121:1283-1299

41. Poulton IJ, McGregor NE, Pompolo S, Walker EC, Sims NA: Contrasting roles of leukemia inhibitory factor in murine bone development and remodeling involve region-specific changes in vascularization. J Bone Miner Res 2012, 27:586-595

42. Braun T, Zwerina J: Positive regulators of osteoclastogenesis and bone resorption in rheumatoid arthritis. Arthritis Res Ther 2011, 13:235 